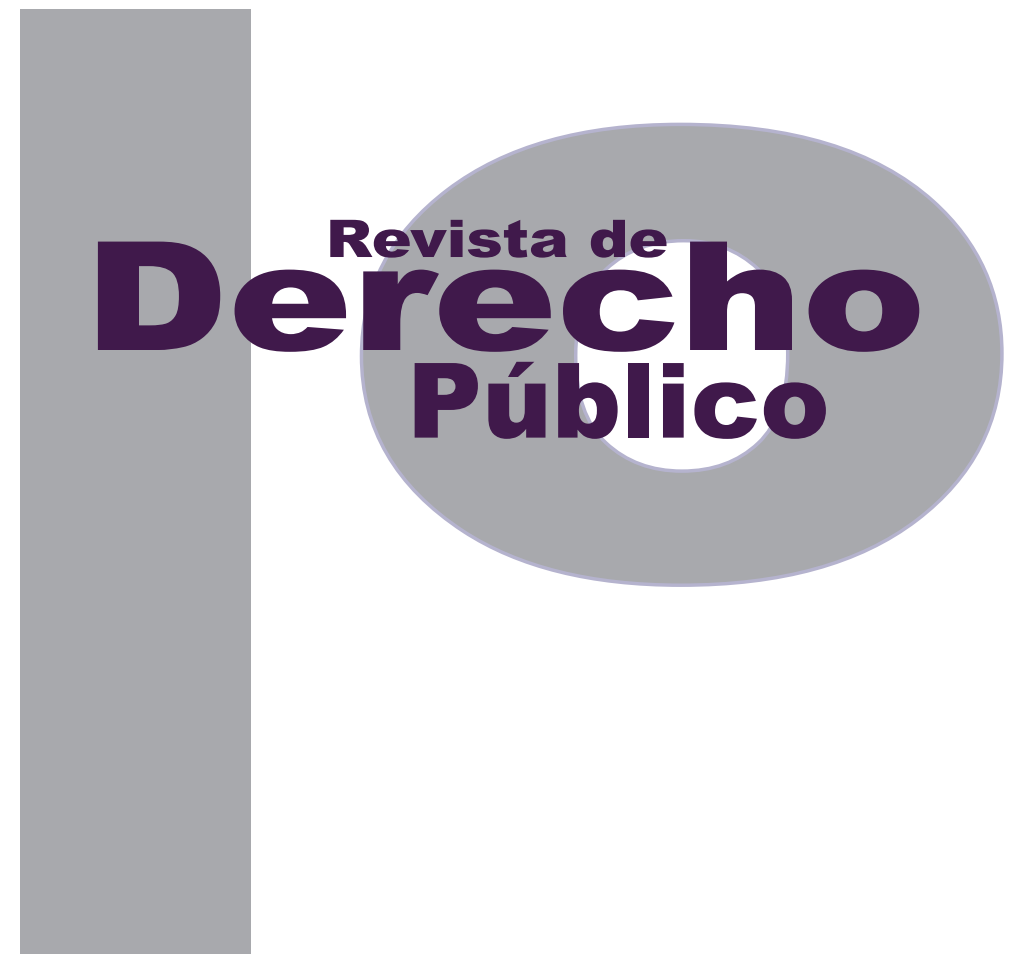

\title{
PRINCIPIO DE EFICIENCIA TRIBUTARIA Y NEUTRALIDAD: INCIDENCIAS TEÓRICAS Y PRÁCTICAS DESDE EL IVA
}

\author{
Lina María Uribe
}

Universidad de los Andes

Facultad de Derecho

Revista de Derecho Público N. ${ }^{\circ} 30$

Enero - Junio de 2013. ISSN 1909-7778 


\title{
Principio de eficiencia tributaria y neutralidad: incidencias teóricas y prácticas desde el IVA
}

\author{
Lina María Uribe*
}

\begin{abstract}
RESUMEN
El principio constitucional de eficiencia en materia tributaria es un concepto con múltiples acepciones, entre las que se encuentra la eficiencia económica vista como la mínima distorsión posible. En el caso del IVA, las características de bases amplias y uniformidad de tarifas satisfacen el principio de eficiencia, entendiéndolo bajo su acepción de neutralidad. No obstante, estas características ideales traen aparejadas objeciones por las cargas que suponen sobre la canasta familiar y por recaer sobre los hogares de menores ingresos de forma más acentuada. Ante el auge e importancia de los impuestos de valor agregado a nivel nacional e internacional se busca observar cómo la eficiencia puede resultar un vehículo hacia un sistema tributario potencialmente redistributivo.
\end{abstract}

Palabras Clave: IVA, impuestos de valor agregado, impuestos indirectos, principio de eficiencia, neutralidad tributaria.

\begin{abstract}
The constitutional principle of tax efficiency is a concept with multiple meanings, where economic efficiency is seen according to its minimal distortion. In VAT, the characteristics of broad base and single rate tariffs satisfy the principle of efficiency, being understood as neutrality. However, those ideal attributes raise objections about the economic burden on a family's daily expenses and the fact it is shown more aggressively in low income households. Facing the increasing importance of value added taxes at domestic and international level, it is intended to observe how efficiency can be understood as a mean which aims for a potentially redistributive tax system.
\end{abstract}

KEY WORDS: VAT, value-added tax, indirect taxation, principle of efficiency, tax neutrality. 


\section{SUMARIO}

Introducción - I. EL PRINCIPIO CONSTITUCIONAL DE EFICIENCIA - A. Principio técnico y equívoco - B. Sentencia C - 776 de 2003: límites a la autonomía del legislador - C. Principio de neutralidad - II. EFICIENCIA ECONÓMICA - A. Características ideales del IVA - B. Inequidad de las condiciones ideales - C. Una distorsión particular: incidencia sobre precios de la canasta familiar - III. IVA: TEORÍA Y PRÁCTICA CONFRONTADA - A. Caso Colombiano - 1. Definición de canasta familiar e incidencia sobre su precio - 2. Sistema de exenciones y exclusiones - B. Contexto internacional de la aplicación de impuestos de valor agregado: práctica en auge - IV. CONCLUSIONES: POSIBLES LINEAMIENTOS - Bibliografía. 
"Capitation taxes are levied at little expense; and, where they are rigorously exacted, afford a very sure revenue to the State. It is upon this account that in countries where the ease, comfort, and security of the inferior ranks of people are little attended to, capitation taxes are very common".

Adam Smith, (1892), Wealth of the Nations.

Introducción

En el año 2003, la Corte Constitucional se pronunció acerca de la exequibilidad de una ampliación del impuesto al valor agregado (IVA) hacia alimentos de consumo diario. Los argumentos a favor señalaban que esta medida aumentaría la eficiencia del IVA. La posición en contra sostuvo que tal disposición contrariaba los principios constitucionales de equidad, punto de vista que finalmente prevaleció en el pronunciamiento de la Corte.

Este debate dista de ser anecdótico, un caso aislado, o de otro modo, de ser un debate superado. El carácter uniforme de la tarifa que se cobra en los impuestos de valor agregado hace que la implementación de estos no permita diferenciar el nivel de ingresos de quien está realizando la transacción. Por ende, objeciones relativas a la equidad al implementar tributos de este tipo surgen de forma acentuada al discutir el gravamen a productos de la canasta familiar, en particular sobre alimentos de consumo diario. No obstan- te, tales oposiciones contrastan con la creciente implementación de impuestos de valor agregado a nivel nacional e internacional.

A la hora de responder si el gasto es un indicador válidamente ético y jurídico de capacidad de pago (Englisch, 2009), y por lo tanto decantarse a favor o en contra de impuestos tales como el IVA, es necesario observar ciertamente el principio de equidad. No obstante, no debe dejarse a un lado otras consideraciones, tales como el principio de eficiencia, que tiene también rango constitucional. Vemos por lo tanto, aquí, una colisión de dos principios rectores del sistema tributario, que puede caracterizarse como un caso difícil, donde el análisis debe intentar realizar una tarea integradora y multidisciplinaria, siendo explícita de cualquier modo la naturaleza no definitiva de estos (Englisch, 2009).

Esta investigación tendrá por objetivo delimitar el contenido e incidencias prácticas del principio de eficiencia en materia de tributación y contrastarlo con rasgos que académicamente se le atribuyen a los impuestos indirectos.

Para desarrollar lo anterior, se dividirá el presente documento en cuatro partes. En primer lugar, se referirá el marco jurídico del principio constitucional de eficiencia. En segundo lugar se caracterizarán, de acuerdo con modelos económicos, las particularidades que se le atribuyen a los impuestos indirectos eficientes, mostrando por un lado qué forma adoptan estas características en materia de tributación y cómo operan las inequidades surgidas de estos. Tercero, se expondrán las incidencias prácticas que la eficiencia en el Iva tiene a nivel de hogares en Co- 
lombia, y posteriormente se comentará el caso de Chile y España. Por último, se concluirá sintetizando los rasgos teóricamente ideales y una posible perspectiva de armonización con estándares de equidad.

\section{EL PRINCIPIO CONSTITUCIONAL DE EFICIENCIA}

El primer paso para discutir el conflicto surgido del IvA respecto a sus posibles características económicamente eficientes consiste en describir cómo el ordenamiento jurídico aborda el principio de eficiencia. Para este fin, se señalarán las acepciones que este principio ha adoptado, en primera instancia. Delimitando estas acepciones, se tratará este principio como parámetro técnico al que acude la autonomía del legislador.

\section{A. Principio técnico y equívoco}

El artículo 363 de la Constitución de 1991 se enmarca dentro del título que regula la configuración económica que surge de la Carta. Con el ordenamiento constitucional, la dirección que tomó la tributación resulta ser un asunto que no solo se limita a la relevancia económica de la hacienda pública: se convierte en una cuestión de trascendencia constitucional. Así, se establece que "El sistema tributario se funda en los principios de equidad, eficiencia y progresividad" (Constitución Política, art. 363).

El segundo de estos principios es el eje principal de este documento. En efecto, se constituye como un fundamento que tiene un significado en materia de tributación menos intuitivo que Ios otros. E incluso, tiene la paradoja de ser considerado como un elemento de alguna forma extraño al texto constitucional. Así, no se trata únicamente de un mandato político, sino que alude más a un "carácter técnico tributario" (Lewin, 2002a, pág. 118), más cercano a la legislación u otro tipo de regulación de inferior jerarquía pero con más vocación práctica.

Lo anterior puede ser un factor que incida en la relativamente escasa discusión al respecto que puede encontrarse, tanto en la doctrina como en la jurisprudencia de altas Cortes que se han pronunciado al respecto (Lewin Figueredo, 2002b). Su inclusión atípica dentro de los principios constitucionales también puede ser un factor que apunte hacia una multiplicidad de ámbitos que se la han atribuido, y que si bien no son totalmente excluyentes sí pueden considerarse diferentes. Esta ha sido una posición que de forma reiterada sostiene la Corte, quien desde mediados de los noventa ha reconocido la existencia de varios significados del principio de eficiencia en tributación:

el citado principio resulta ser "un recurso técnico del sistema tributario dirigido a lograr el mayor recaudo de tributos con un menor costo de operación; pero de otro lado, se valora como principio tributario que guía al legislador para conseguir que la imposición acarree el menor costo social para el contribuyente en el cumplimiento de su deber fiscal (gastos para llevar a cabo el pago del tributo) ${ }^{1}$.

1 Corte Constitucional. Sentencia C-198 de 2012. M. P.: Nilson Pinilla. 
La anterior cita permite delimitar al menos tres formas que puede adoptar el principio de eficiencia. La primera resulta de contrastar el beneficio y el costo en que incurrió el Estado al implementar el tributo; remite entonces más a la correcta racionalidad de la hacienda pública en la consecución de recursos para la realización de fines que le son propios, cercana a la efectividad del recaudo. Una segunda forma que es posible observar se relaciona no con el Estado, sino con el contribuyente: debe existir moderación sobre los gastos que se le imponen a este para cumplir con la obligación tributaria. Los recursos necesarios, tanto para ejercer la posición activa como pasiva de la obligación tributaria parecen ser el principal interés de la Corte Constitucional y el Consejo de Estado al pronunciarse sobre el principio de eficiencia ${ }^{2}$. En todo caso, se considera que estos han actuado más como complementos de su argumentación que como ejes centrales a esta (Lewin Figueredo, 2002b)

No obstante, a partir de los costos sociales también se abre la puerta a una tercera forma que puede adoptar el principio de eficiencia, que es la que resulta pertinente a la discusión que se presenta. Se define entonces eficiencia no como una referencia a "el manejo y administración misma de los tributos por parte de las autoridades hacendísticas, sino a sus efectos en la economía en general" (Plazas Vega, 2006, pág. 433). Tal modalidad invita a un análisis adicio-

2 Ver también: Corte Constitucional, sentencia C-397 de 2011, M. P.: Jorge Ignacio Pretelt Chaljub; Corte Constitucional, sentencia C-261 de 2002, M. P.: Clara Inés Vargas; Consejo de Estado, Sección Cuarta, sentencia Rad. 11001-03-27-000-2007-00048-00 (16873) del 26 de noviembre de 2009, C. P.: Héctor Romero Díaz. nal a la hora de seleccionar cuáles cargas se impondrán y cuál nivel adoptarán estas, puesto que ahora se deberá observar también sus efectos.

Ciertamente, existen diferentes formas de acercarse a los efectos de la política tributaria, y distintas maneras de proveer un juicio normativo que califique qué situación resulta mejor. La literatura académica del análisis económico del derecho ha contemplado varios enfoques. Un primer método para tal evaluación ha sido decidir a través de un enfoque utilitarista: observar si la mayoría de individuos se ven beneficiados (Rubio, 2007). Tras las objeciones que surgen al aplicar tal aproximación en una democracia que supera una dimensión cuantitativa, como es el caso de un Estado social que cuenta entre sus fundamentos la solidaridad de sus ciudadanos, se hace necesario pensar en otros criterios. Un enfoque recurrente ha sido analizar la eficiencia por vía de quiénes ganan o quiénes pierden al adoptar una decisión. Tal criterio ha remitido a la idea de óptimo de Pareto, bajo la cual se considera una situación como óptima si se logra mejorar al menos a una persona sin desmejorar a otra (Roemer, 2008). Las limitaciones que este enfoque presenta inician con las dificultades empíricas de encontrar una situación que genere un resultado positivo o neutro para cualquier caso. Por lo tanto, ante el reconocimiento de la existencia de ganadores y perdedores a partir de determinada norma o institución jurídica, se amplió con el concepto de óptimo de Kaldor-Hicks. Ante la polaridad entre perdedores y ganadores que puede surgir, en este caso a raíz del impuesto de valor agregado, existe una 
potencial forma de valorar una situación como globalmente benéfica si los ganadores están en capacidad de compensar a los desfavorecidos con la introducción de la medida. De esta manera, se encuentra una intersección que armoniza la eficiencia y generación de riqueza con criterios de distribución y cierta justicia social (Rubio, 2007).

En síntesis, este principio, entendido como los efectos económicos de la tributación, ha sido considerado un recurso técnico con el cual cuenta el legislador. Adicionalmente, este puede en principio escoger qué tipo de efecto desea imprimir con el impuesto, "siguiendo su propia evaluación, sus criterios y sus orientaciones en torno a las mejores conveniencias de la economía y de la actividad estatal" ${ }^{34}$.

\section{B. Sentencia C - 776 de 2003: límites a la autonomía del legislador}

Habiendo observado que existe determinada libertad de configuración respecto al impacto que previsiblemente tenga el impuesto, también se ha manifestado la Corte en el sentido que esta libertad está supeditada al respeto de otros principios. Para esta investigación, se expondrá dentro de la definición de eficiencia un conflicto particular que surge en el marco de los impuestos indirectos. El aparente antagonismo entre equidad y eficiencia en impuestos tales como el Iva es un tema que discute la Corte en

3 Corte Constitucional, sentencia C-198 de 2012, M. P.: Nilson Pinilla.

4 Ver: Corte Constitucional, sentencia C-341 de 1998, M. P.: José Gregorio Hernández; Corte Constitucional, sentencia C-222 de 1995, M. P.: José Gregorio Hernández. la sentencia 776 de 2003. El pronunciamiento analiza la constitucionalidad de la Ley 788 de 2002, donde se contempla la ampliación de la base como una forma de eliminar la fragmentación y heterogeneidad normativa en materia tributaria. En su artículo 116 imponía una tarifa de $2 \%$ a los bienes excluidos hasta ese momento en el Estatuto Tributario (Art. 424), artículo que fue demandado y posteriormente declarado inexequible.

La posición de la Corte muestra una situación donde la autonomía del legislador, al decidir sobre la eficiencia del tributo, encuentra un claro límite en el mínimo vital. Reconoce en este pronunciamiento que un análisis de contexto económico del impuesto es un deber necesario para el legislador. No obstante, al hablar de la imposición de un gravamen de $2 \%$ sobre alimentos "se torna relevante no solo el derecho a la vida sino otros derechos sociales, como la salud, de los cuales depende el goce efectivo del primero" ${ }^{5}$. Estos derechos se condensan así en el concepto de mínimo vital, que reune condiciones materiales sobre elementos tales como alimentación, vivienda o ropa, que aseguran una vida digna (Arango, 2005).

El mínimo vital, a juicio de la Corte, se pone en riesgo con la expansión de la base gravable. Y esto, junto con el hecho de no haber contado con una deliberación suficiente en el Congreso, ni con medidas compensatorias de esta pérdida de poder adquisitivo, es uno de los argumentos que esgrime la Corte para declarar la inexequibi-

5 Corte Constitucional, sentencia C-776 de 2003, M. P.: Manuel José Cepeda. 
lidad de la medida. Esta posición, que pondera la equidad y la eficiencia en el IVA, se observa de nuevo en el 2010, cuando la Corte reitera el mínimo vital, en tanto que elemento de la equidad, como un límite importante para la delimitación de la base gravable del IvA ${ }^{6}$.

No obstante, el tema no está zanjado. Incluso, la Corte manifiesta en ocasiones anteriores la conformidad de impuestos indirectos con el ordenamiento constitucional, aún cuando "por la conformación técnica de ese tributo indirecto y la necesidad de su administración eficiente" 7 genera obligaciones uniformes sin evaluar el caso particular del que está contribuyendo. Y en la sentencia C-776 de 2003 la Corte afirma claramente que la imposición sobre bienes de primera necesidad no es per se inconstitucional. De la sentencia se desprende que es viable la discusión sobre ampliar la base del IvA siempre y cuando se respeten los canales de deliberación democrática, y cuando "existan políticas efectivas que compensen la afectación al mínimo vital de las personas que, debido a su condición económica, enfrentarían dificultades"8.

Afirmar que es factible complementar la creación de nuevas obligaciones tributarias con medidas que aseguren un bienestar mínimo no es una idea aislada en la Corte. Y de otro modo, no constituye un disenso frente a opiniones de académicos de diversas áreas quienes se encuen-

6 Corte Constitucional, sentencia C-173 de 2010, M. P.: Jorge Ignacio Pretelt Chaljub.

7 Corte Constitucional, sentencia C-597 de 2000, M. P.: Álvaro Tafur.

8 Corte Constitucional, sentencia C-776 de 2003, M. P.: Manuel José Cepeda. tran de acuerdo en que al momento de evaluar la equidad del sistema tributario es imprescindible observar "el destino del gasto público" (Ukmar, 2002, pág. 72). Así, la equidad no solo se predica de las formas en las que el Estado exige las obligaciones tributarias, sino que también se debe contemplar el destino que el Estado da a los recursos recaudados. Si estos recursos son implementar acciones dentro de una política social enfocada en los sectores más pobres, no habrá lugar a considerar un sistema tributario como inequitativo.

Con posterioridad a la Ley 788 de 2003 no hubo reformas que ampliaran la base del IVA en la magnitud que esta norma propuso. La reforma que hoy se tramita en el Congreso, proyecto de ley 166 de 2012, continúa en la línea de mantener tarifas diferenciales y respecto al conjunto de bienes catalogados como excluidos no introduce cambios sustanciales. En relación con los bienes a los que se les computa una tarifa reducida, el proyecto de ley en su artículo 48, que modifica el artículo 468-1 del Estatuto Tributario, incluye de forma notoria una amplia gama de insumos, almacenamiento, maquinaria y aseguramiento destinados a la producción agrícola. La tarifa reducida sería modificada también, pasando del $10 \%$ al $5 \%$. Adicionalmente, amplía el conjunto de bienes exentos (Art. 52 del proyecto, 476 del Esatuto Tributario), incluyendo en particular alimentos piscícolas. En todo caso, la innovación más importante dentro de la proyectada reforma la constituye la implementación de un impuesto al consumo que recaerá sobre bienes finales pertenecientes a tres clases de productos (Art. 60 y ss). En primer lu- 
gar, sobre el consumo en restaurantes, bares y similares, que estarán excluidos de Iva pero pagarán ahora un impuesto de $8 \%$ sobre el precio final. Los teléfonos celulares deberán pagar un $4 \%$ del precio del servicio, sin contar lo que se cause por concepto de IVA. Y por último, se impone este recargo sobre los bienes considerados de lujo, a los que se cobrará una tarifa del $8 \%$ o $20 \%$ dependiendo del caso.

El objetivo de esta investigación es continuar en la línea de discusión de la sentencia, delimitando el contenido del principio constitucional de eficiencia. Habiendo observado los pronunciamientos de la Corte sobre este y reiterando su jerarquía constitucional, se mostrará el principio de neutralidad como una posible forma de dar contenido a la acepción de eficiencia que describe el impacto económico del tributo.

\section{Principio de neutralidad}

Esta sección describirá el principio de neutralidad como una forma de integrar la eficiencia en el sistema tributario.

Haciendo una síntesis de la exposición de las secciones anteriores, los principios constitucionales que deben guiar el sistema tributario son: equidad, eficiencia y progresividad. No obstante, al intentar dilucidar el conflicto que se ha tejido en torno a la equidad y eficiencia en el tema de tributación indirecta, nos encontramos que la definición mayormente compartida de la segunda muchas veces se asimila más a la efectividad en el recaudo. Esta acepción está en contraposición con otra que es, en general, compartida por los economistas y está referida a un mercado que cumpla con las condiciones de competencia perfecta, donde la oferta y demanda se encuentren por vía de precios que reflejen únicamente su escasez relativa.

Es esta última acepción la que en este trabajo se tiene como propósito examinar con más profundidad. Ante las formas difusas a las que se aproxima el sistema jurídico colombiano a la hora de discutir sobre eficiencia tributaria, resulta pertinente ver cómo se ha entendido el concepto de eficiencia requerida en otros campos. Para esto se acudió a instituciones de investigación, como el International Bureau of Fiscal Documentation (IBFD) y otras instancias como el Tribunal Europeo de Justicia (Dale \& Nieuwenhuizen, 2009) para complementar el contenido de este principio constitucional. Así, encontramos el principio de neutralidad como un marco conceptual que aterriza las características económicamente deseables dentro de los impuestos indirectos como el IVA. Este principio remite a la mínima incidencia que debería tener el impuesto sobre el mercado interno: no interferir ni con las decisiones del consumidor ni con las del productor (Cnossen, 2009).

A partir de las distintas formas en que puede incidir la política tributaria se han conceptualizado las facetas que adopta el principio de neutralidad. Para efectos ilustrativos se presentan las citadas en Nerudova \& Siroki (2009) para el IBFD. Entre estas se encuentra:

Neutralidad jurídica: explica la igualdad en la proporción que grava el producto.

Neutralidad competitiva: busca evitar que la carga impositiva dependa de las fases de pro- 
ducción por las que ha pasado el producto, o el nivel de integración vertical u horizontal del proveedor. Esto, por ejemplo, en sistemas de impuestos sobre las ventas (retail sales) se profundiza cuando no hay descuentos sobre impuestos pagados sobre insumos (Cnossen, 2009). Por lo tanto, a mayor nivel de integración del productor en relación con la fase de producción, menos cargas tributarias pesarán sobre el, pudiendo fijar un menor precio u obteniendo un mayor margen de ganancias.

Neutralidad externa: indicativa de cuánto puede estar favorecido o no el comercio internacional de un país según su tasa de impuestos. Por ejemplo, cuando un bien importado se grava de forma más onerosa que un bien producido a nivel nacional, se estaría violando el principio de neutralidad externa, pues los productos nacionales tendrían ventajas frente a su contraparte importada.

Neutralidad económica. Esta modalidad resulta la más importante para efectos de la discusión. "La neutralidad económica está definida en estrecha conexión con la asignación eficiente de los recursos producidos. Para asegurar la asignación eficiente de recursos, las tarifas del impuesto deben influenciar los mecanismos de mercado en el menor grado posible". (Nerudova \& Siroki, 2009, págs. 222, traducción propia).

En consonancia con lo que se ha explicado, el impuesto debe generar una situación lo más cercana posible a la existente antes de su imposición. En particular, como lo evidencia la cita, se hace énfasis en la forma en que influye sobre los mecanismos de mercado. Entre estos, el precio es central, pues en efecto es uno de los canales más importantes para la asignación de los bienes que se están transando. Dependien- do de cuánto sea el precio de estos y de cuánto son valorados por los consumidores, estos terminarán por decidir qué comprarán.

Por lo tanto, un cambio en los precios, ciertamente, cambia también la asignación de bienes. En esto hay dos tipos de cambios. El nuevo precio puede, por un lado, generar sencillamente un menor consumo y, por otro, alterar las proporciones entre los precios de los productos. Esto resultará en que el consumidor varíe la cantidad que consumía de cada uno en forma diferente.

Estos dos cambios regresan la discusión a los conceptos expuestos en el numeral anterior. Así, el efecto ingreso se relaciona precisamente con el menor ingreso disponible que resulta de los mayores precios. En el caso de los impuestos como el IVA, las menores posibilidades de consumo generadas por el cobro del precio más la fracción cobrada como gravamen.

El efecto sustitución se relaciona, por su parte, con cambios en los comportamientos de los consumidores con el fin de aligerar la carga tributaria que deben pagar. Como se mencionó anteriormente, estos pueden incidir en la proporción consumida de diferentes bienes, como consecuencia de la percepción de qué tan barato es uno frente a otro. No obstante, hay otras vías por las que se puede evidenciar el efecto sustitución. Por un lado, el consumidor puede decidir reducir el tiempo que trabaja, si observa que proporcionalmente está pagando más impuestos sobre su salario y lo que puede ahora consumir es mucho menor. Pero puede adicionalmente decidir que una opción más conveniente es seguir consu- 
miendo estos bienes, pero en el mercado informal. (Nerudova \& Siroki, 2009).

Este efecto sustitución termina siendo el criterio definitivo a la hora de calificar si un impuesto es neutral o no. Todo impuesto, necesariamente, afecta las posibilidades de consumo de un contribuyente (genera un efecto ingreso). Si recae directamente sobre el ingreso, como el impuesto de renta, disminuye el dinero disponible. Si se trata de un impuesto indirecto y recae sobre el consumo, como el IVA, el contribuyente se enfrenta a un mayor valor de los bienes. No obstante, se considerará como neutral aquel impuesto que no crea cambios adicionales en los comportamientos de los consumidores y que, por lo tanto, no tiene efecto sustitución.

En este punto atamos lo expuesto sobre eficiencia en el numeral anterior, con lo expuesto aquí sobre neutralidad. ¿De qué forma? La inexistencia de efecto sustitución que requiere el principio de neutralidad coincide entonces con la visión de minimización de distorsiones. Por consiguiente, puede cerrarse la discusión fijando el principio de neutralidad económica como una manera de conceptualizar, desde la teoría tributaria, la minimización de distorsiones, afirmación que ya otros autores han propuesto al hablar de tributación normativa (Cullis \& Jones, 1998). Este, por lo tanto, permite ser un punto de referencia entre los requerimientos teóricos de la economía que se señalarán a continuación frente a las categorías jurídicas propias del derecho tributario, como lo es el principio constitucional de eficiencia.

\section{EFICIENCIA ECONÓMICA}

Ahora, para caracterizar el principio de eficiencia en el IVA desde un punto de vista económico, se describirán sus características ideales y se expondrá cuáles son las principales incidencias que tendrá este recaudo a nivel de distorsión en el mercado. Para tal fin esta sección mostrará las principales condiciones ideales que describen distorsiones económicas mínimas, y, por último, se hablará del caso particular de la relación entre IVA y precios como una manifestación de estas distorsiones.

\section{A. Características ideales del IVA}

Es pertinente iniciar esta exposición explicando dos generalidades importantes del IVA, en cuanto impuesto indirecto, para responder posteriormente qué rasgos deben tener estos para alcanzar un determinado nivel de eficiencia.

En primer lugar, el IVA posee un carácter indirecto: el Estado se vale de lo que una persona consume para tomarlo como manifestaciones presuntas de riqueza (Englisch, 2009, pág. 1, traducción propia). Teniendo en cuenta que se cobra a todo aquel que realice una transacción se puede observar que es muy amplio el rango de personas sobre el que recae. Por esta misma característica de impuesto indirecto se torna impersonal, debiéndose abstraer de las condiciones particulares de quien está realizando las transacciones gravadas. Se enfrentan aquí dos ideas: al tiempo que establece un patrón de absoluta igualdad en cuanto a lo que cada uno está pagando, imposibilita establecer cuál es la 
contribución justa y proporcional que debería realizar cada persona que está consumiendo (Hemels, 2009). Esto se contrapone a la situación que se presenta en los impuestos directos que recaen sobre el ingreso, tales como el impuesto de renta. En ellos la tarifa puede adecuarse de manera proporcional al ingreso de la persona que está tributando, siendo además posible observar de forma clara cuál es la capacidad de pago. Esto incide, entre otras cosas, en la calificación de los impuestos directos como no regresivos (Moller, 2012).

Habiendo establecido tales características del IVA como impuesto indirecto, impersonal, se responderá ahora cuál es el ideal que se le ha atribuido a los impuestos indirectos. Es decir, ¿qué características se han definido como necesarias para que este impuesto sea calificado como eficiente?

Se describirán dos rasgos que algunos modelos académicamente reconocidos le atribuyen a los impuestos indirectos: bases gravables amplias y cobro sobre productos inelásticos. Este segundo rasgo se definirá sucintamente para entender lo que se considera un bien inelástico.

El primer rasgo, que la base gravable sobre la que recae debe ser lo más extensa posible, significa que la lista de productos cuyo precio incluya el Iva debe, por su misma amplitud, reducir las distorsiones existentes. La lógica detrás de esto podría pensarse de la siguiente forma: si absolutamente todos los bienes están gravados con la misma tarifa, el contribuyente termina pagando igual fracción por cada bien que consume, con lo cual aporta esa fracción sobre su ingreso
(Fergusson \& Suárez, 2010). En otras palabras, si una persona consume todo su ingreso y paga $16 \%$ por todos los bienes que adquiere, al final estará tributando un 16\% sobre su ingreso. Una base amplia, por lo tanto, hace que el impuesto al consumo reduzca las diferencias que podría haber antes y después de recaudar el impuesto. Teóricamente el impuesto que abarca una base amplia lo único que hará será reducir la escala, sin afectar las proporciones entre cantidades y precios de un bien sobre otro.

Un segundo rasgo es su cobro sobre los bienes más inelásticos. Estos son aquellos cuyo consumo se afecta en menor grado por variaciones en el precio, en razón de que se consumen en una cantidad fija, independientemente de cuánto cuesten. Usualmente se identifican con productos o servicios de primera necesidad, en oposición a otros bienes accesorios o suntuarios de los que, ante incrementos acentuados en su precio, puede prescindirse. La relación que existe entre esta definición y su inclusión como un impuesto eficiente se encuentra en que el mayor precio, proveniente del cobro del IVA, afectará lo mínimo posible la decisión de una persona sobre qué consumir.

Bajo la óptica de la búsqueda de eficiencia, si lo que se quiere es minimizar los cambios en las decisiones de los consumidores, un impuesto que grave el precio repercutirá menos si recae sobre un bien que estos estén menos dispuestos a dejar de comprar que si lo hace sobre uno que puede resultarles accesorio y, por tanto, no tendrían problema en no adquirirlo. El consumidor terminará gastando más en el producto que está de alguna forma obligado a comprar, y en 
términos generales su mercado no cambiará, con lo cual genera un amplio recaudo. No obstante, la situación del consumidor empeora por vía de este mayor gasto, que económicamente se expresa en la pérdida de bienestar. Al crearse un tributo sobre productos inelásticos el resultado no será un menor consumo, por el contrario, dado que recae sobre bienes que se consumirán de forma más o menos fija, lo que ocurrirá con los hogares será que se verán obligados a pagar el nuevo precio (Jaramillo \& Tovar, 2007a).

\section{B. Inequidad de las condiciones ideales}

Describimos anteriormente las características de amplitud de la base y gravamen sobre bienes inelásticos que los modelos plantean como objetivos que deben ser alcanzados. Ahora llegamos a la discusión sobre cómo los ideales de eficiencia no tienen una relación evidente con la equidad, y cómo tales limitaciones inciden en los hogares, en particular sobre su ingreso disponible y sus preferencias.

Los dos rasgos señalados empiezan a vislumbrar las posibles objeciones. Cuando la clave de su eficiencia teórica radica en la implementación de una base amplia o en el gravamen de bienes inelásticos, la discusión sobre su equidad retoma fuerza. Por el lado de una base amplia se alcanza una gama de productos mayor que cubre aquellos de la canasta básica. Y por el lado de los bienes inelásticos, es evidente que un impuesto que basa su eficiencia en la disminución del bienestar del consumidor abre un camino propicio para objeciones relativas a su conveniencia en el marco de un Estado social. Y si se deseara gravar bienes inelásticos, se ten- dría que considerar gravar productos como los de la canasta familiar.

Estos problemas se ven reflejados de forma más aguda en los hogares de menos ingresos, teniendo en cuenta que la carga del impuesto sobre una base amplia cubre productos de carácter necesario, tales como los alimentos, a los que se aplica la misma tarifa de impuesto independientemente del nivel de ingresos de la persona. Por lo tanto, el valor del impuesto sobre estos productos absorbe una fracción mayor de un ingreso bajo que de uno alto, afectando en mayor grado a los hogares de bajos ingresos. Puede pensarse en dos hogares, uno de ingresos bajos y otro de ingresos altos, que consumen una cantidad similar de alimentos y, por lo tanto, destinan una suma de dinero equivalente a alimentos. Sin embargo, aún cuando la suma de dinero sea la misma, la fracción resulta ser mayor en el hogar de ingresos bajos que en el hogar de ingresos altos. Cuando se aplica una tarifa uniforme de IVA sobre estos productos, en un razonamiento análogo, el pago de este impuesto termina requiriendo una fracción mayor del ingreso bajo que del ingreso alto.

En los hogares de bajos ingresos se desdibuja la definición de impuestos indirectos, vistos como manifestaciones presuntas de riqueza (Englisch, 2009, pág. 1, traducción propia). En el consumo de productos necesarios no se puede hablar de manifestaciones de riqueza, sino de transacciones que se limitan al mantenimiento de un nivel mínimo de calidad de vida. Las condiciones ideales muestran, aun desde una perspectiva puramente teórica, que la tributación indirecta plantea de entrada un ejercicio de equilibrio 
entre corrientes contrapuestas. La paradoja es, de una u otra forma, que los diseñadores de la política tributaria deben acudir a la tributación indirecta. Incluso si desean imprimirle un mayor contenido redistributivo, necesitan implementar o continuar aplicando impuestos indirectos, a sabiendas de las limitaciones en equidad que implica el modelo de base amplia y gravamen sobre bienes inelásticos.

A juicio de algunos, la tributación directa (impuestos tales como el de renta) puede resultar ser un instrumento idóneo para mantener determinado recaudo, controlando al mismo tiempo criterios de proporcionalidad y eficiencia. El gravamen sobre el ingreso de forma escalonada resulta ser, en efecto, una política redistributiva: los ciudadanos de mayores ingresos deben tributar un porcentaje mayor sobre estos, los cuales son, además, perfectamente visibles para las autoridades a partir de la declaración de renta y documentos similares. Esto, por el contrario, no es posible en el cobro del IVA: no hay forma de cobrar una tarifa de acuerdo con el nivel de ingreso que tiene el consumidor.

No obstante, existen razones relativas al fomento de la generación de riqueza que recomiendan evitar el excesivo gravamen de la renta o ingresos, por lo que es imposible depender exclusivamente de la tributación directa. Teniendo esto en cuenta, se llega a la decisión de escoger "el segundo mejor" (Atkinson, 1977, pág. 14, traducción propia): una opción que atienda las restricciones prácticas y teóricas del problema de impuestos indirectos vs. directos aunque cierta-

9 “Second- best” (Atkinson, 1977, pág. 14) mente no cumpla con la definición estricta de óptimo. Alternativa que, en todo caso, se muestra como la segunda mejor entre las posibles. Por lo tanto, es necesario partir de antemano de que habrá impuestos indirectos, y que forzosamente tendrán incidencia sobre los hogares en dos niveles: ingresos y preferencias.

En ingresos, porque el dinero disponible de los hogares cambia con el impuesto como consecuencia de la fracción de tributación sobre el precio de lo que consumen, suma con la que, de otro modo, el hogar podría contar. En este sentido, el poder de compra que pierde el hogar frente a los nuevos precios que resultan del IVA es lo que recibe el nombre de efecto ingreso. $Y$ como se expuso anteriormente, esto constituye de entrada una pérdida de bienestar para el hogar (Jaramillo \& Tovar, 2007a).

En cuanto a preferencias, la existencia de bienes que tengan tarifas cambiará la forma como perciba el consumidor que un precio es más costoso, proporcionalmente, que otro. Lo que ahora se debe tener en cuenta es la reducción del cambio entre las relaciones de proporción de los precios, dado que contemplará el cobro de distintas tarifas de IVA sobre estos. Estos cambios en las relaciones de precios - qué tan costoso resulta un bien si se observa la proporción entre su precio y el de otro- reciben el nombre de efecto sustitución. Por lo tanto, el principal margen en el que se debe mover es en el de la minimización de estos cambios en precios relativos, que se tienen como distorsiones.

Anticipando lo que se explicará con posterioridad, este objetivo de minimización de distorsio- 
nes es, en efecto, de interés para los hogares, a pesar de parecer una variable que solo se encuentra en la literatura pertinente. El objeto de este proyecto es delimitar la contraposición entre equidad y eficiencia. Aunque es cierto que la equidad podría considerarse un elemento de interés de todos los hogares, y en particular de los más vulnerables, no por esto el problema de minimización de distorsiones resulta irrelevante para estos. El tema al que conduce esta discusión es la forma como esta segunda mejor opción ha sido englobada, para el caso particular de la tributación indirecta, bajo el principio de neutralidad.

\section{Una distorsión particular: incidencia sobre precios de la canasta familiar}

Teniendo la neutralidad como punto de referencia en la discusión sobre el principio de eficiencia, se pasa a explicar brevemente la relevancia de la controversia.

Recapitulando: previamente se mencionó que en la línea de la sentencia 776 de 2003 existe una oposición entre equidad y eficiencia con respecto a la tributación indirecta. No obstante, habría que preguntarse si, por lo demás, tal oposición resulta ser una disertación puramente teórica. La respuesta a esta pregunta es no. Este conflicto, más allá de tener una descripción académica, es un asunto presente en la cotidianidad.

El impuesto indirecto se transfiere al consumidor: este es sobre quien recae la suma extra de dinero reflejada en los precios (Nerudova \& Siroki, 2009). Por lo tanto, el IVA resulta ser un impuesto omnipresente, dada su existencia en una infinidad de transacciones. Y cuando el problema se centra en productos de consumo diario, pertenecientes o no a la canasta familiar, es posible derivar que la discusión es plenamente relevante. Así, al hablar de las múltiples transacciones sobre los productos de primera necesidad cuyo precio puede aumentar a causa del IVA, resulta pertinente la disquisición sobre su eficiencia que, valga aclarar, no será siempre automáticamente antagónica de la equidad.

A continuación se expondrán las diferentes formas en que la eficiencia incide efectivamente a nivel nacional e internacional.

\section{IVA: TEORÍA Y PRÁCTICA CONFRONTADA}

Parte de los objetivos que se presentaron es señalar de qué forma el Iva tiene incidencia en la vida cotidiana de sus contribuyentes. Para esto, se intenta integrar parte de la discusión teórica expuesta en el acápite anterior con lo señalado referente al marco constitucional y legal del IVA.

Por lo tanto, se pasará a analizar qué prácticas se han desarrollado a nivel colombiano. Posteriormente se expondrá el contexto en el cual se ha implementado este impuesto a nivel internacional y, por último, se describirán casos de implementación relevantes para Colombia.

\section{A. Caso colombiano}

En esta sección se plasmarán los efectos concretos del gravamen del IVA en tres aspectos: en primer lugar, aquel relacionado con bienes 
de la canasta familiar; en seguida el tema de la potencial pérdida de eficiencia resultado de la regulación derivada de exenciones y exclusiones; y, finalmente, el escenario que plantea la eventual entrada en vigor de la regulación de la Comunidad Andina de Naciones.

\section{Definición de canasta familiar e incidencia sobre su precio}

Respecto de los efectos sobre precios, es necesario plantear ahora algunas definiciones relativas al objeto sobre el que estos recaen.

Una definición que resulta clave es la de canasta familiar. Esta es entendida como un conjunto de bienes y servicios que se consideran inherentes al desarrollo normal de un hogar y, por lo mismo, son demandados por cualquier hogar. Esta tiene como corolario que no solo remite a los bienes o servicios que puedan ser adquiridos con un salario mínimo: intenta ser, ante todo, una "canasta para seguimiento de precios" (Dane, 2012). Los requisitos para la inclusión de un bien en tal conjunto de productos comprende el análisis de si este tiene una alta participación en el consumo de los hogares o si es demandado por la mayoría de ellos (más del treinta por ciento de estos (Lora, 2008)). Además, si es producto que tiene o se prevé que tenga una tendencia creciente en el gasto a mediano o largo plazo.

Vale la pena recordar que no solo contiene servicios de primerísima necesidad. También comprende otros no tan íntimamente ligados a una subsistencia física que intuitivamente remiten a los alimentos de primera necesidad. No obstante, esto no les quita su calidad de bien o servicio de gran impacto para los hogares. Así, el análisis de lo que es constitutivo de la canasta familiar incluye ocho grupos de gasto: alimentos, vivienda, vestuario, salud, educación, cultura, transporte, comunicaciones, diversión y esparcimiento, y otros (Lora, 2008). Como ejemplo pueden mencionarse computadores, elementos de estudio, y otros servicios que no se asocian con la supervivencia física de un hogar, pero que en todo caso resultan claves para la calidad de vida y el normal transcurso de los hogares.

Esta definición no solo ofrece una ilustración marginal. Una parte del problema relacionado con la aplicación del IVA a productos de la canasta familiar está en evaluar cuáles productos entran y cuáles no.

En efecto, el filtro que define los bienes y servicios esenciales para un hogar no tiene una capacidad perfecta para describir qué es necesario y qué es suntuario. Además, no es inmutable y puede incluir determinado grado de subjetividad a la hora de establecer un conjunto final. El Dane, entidad encargada de proporcionar la lista oficial de estos productos, la revisa cada cierto lapso de tiempo, analiza cuáles corresponden a uno u otro estatus y evalúa la ocurrencia estadística de los criterios mencionados anteriormente: participación actual o prevista en el gasto, qué tantos hogares consumen tal producto, etc.

¿Qué relación tiene la definición cambiante de la canasta familiar con el conflicto de eficiencia y equidad? Retomamos nuestro problema inicial: el conflicto de eficiencia y equidad al momento de gravar la canasta familiar con el impuesto de 
IVA. Al respecto es necesario precisar que los productos de la canasta familiar tienen un espectro más amplio del intuitivamente analizado, para evaluar el impacto del IVA en términos de equidad no solo por cuenta de alimentos de consumo diario.

En todo caso, delimitar la canasta familiar ya ha sido descrito como un paso clave en el proceso de evaluar qué tanta equidad y eficiencia desea imprimírsele a la tributación indirecta. Imprecisiones respecto de qué se considera necesario generará que productos análogos reciban cargas tributarias diferentes, incurriendo en las citadas distorsiones. Otra dirección en la que la definición de este conjunto de bienes será crucial es en el impacto positivo o negativo que se desea producir sobre determinados bienes y servicios. Así, la inclusión de productos con mayores grados de subjetividad como bienes de la canasta familiar y sin tarifas diferenciales, tales como los bienes culturales, podrían verse perjudicados (Hemels, 2009), a pesar de que su uso o consumo sea considerado positivo y se quiera favorecer de forma global.

Por lo tanto, la delimitación de estos bienes es un primer ejercicio en el que se materializa la disyuntiva entre equidad y eficiencia, pues asegura la inclusión de bienes consumidos por una porción mayoritaria de los hogares. Al mismo tiempo, es una balanza donde se contrapone el interés estatal en la tributación frente al potencial detrimento del consumo deseado de ciertos bienes y servicios.

Habiendo expuesto qué se tendrá como canasta familiar y una parte de la problemática que relaciona la tributación indirecta con esta definición, se pasará al tema de los precios de los productos relevantes. Así, se explicará en este acápite parte de lo que artículos e investigaciones académicas han encontrado respecto a qué variación tienen los precios de bienes consumidos por la gran parte de los hogares.

Un estudio pertinente e ilustrativo puede verse en el ejercicio llevado a cabo por Jaramillo \& To$\operatorname{var}(2007 a$ y $2007 b)$.

En el primer documento los autores estiman la relación que existe entre la tarifa del Iva y lo que los hogares consumen, midiendo esta última variable por medio de cuánto gastan los hogares con o sin impuesto. Tal análisis tiene ciertas limitaciones: no se realiza a toda la canasta familiar, sino a determinados grupos. No obstante, conserva su importancia en la medida que los cuatro grupos de bienes escogidos pueden considerarse representativos (alimentos, por ejemplo); incluye bienes de consumo habitual, tales como productos relacionados con educación, cultura e incorpora el rubro de celulares. En el segundo documento realizan un procedimiento análogo respecto a los precios de la canasta familiar entera, midiendo cuánta ha sido su variación contabilizada por medio del IPC.

Ahora, ¿cuáles son las conclusiones a las que llegan estos estudios? Para empezar, afirman que si bien se encuentra un impacto diferenciado por ciudades, en todas las poblaciones de la muestra se presenta de forma consistente un aumento, tanto en los precios de los productos como en el gasto de los hogares. 
En segundo lugar, concluyen que el impacto del gravamen dista de ser un efecto teórico: de los 146 bienes de la canasta que vieron modificadas sus tarifas de imposición en el periodo de análisis, el 40\% de estos tuvieron un aumento en sus precios en una magnitud de consideración. Entre los precios más susceptibles de variar se encuentran la alimentación por fuera del hogar, servicios financieros, telefonía, ropa infantil, entre otros (Jaramillo \& Tovar, 2007b).

Hay que mencionar que los productos hoy exentos no vieron modificada su tarifa de 1998 a 2006 y, por lo tanto, el estudio de estos autores no puede extenderse a cuál sería el impacto de la introducción de una tarifa sobre ellos. No obstante, refuerzan lo afirmado en el acápite anterior sobre cómo la definición de productos de primera necesidad resulta ser un paso crucial. En efecto, actividades que podrían pensarse como positivas se ven afectadas en gran medida, tales como el acceso a servicios financieros o a telefonía y comunicaciones.

Las conclusiones respecto al gasto reiteran que la tributación es un problema con claras incidencias en materia de equidad. El análisis de Jaramillo \& Tovar (2007a) indica, en primer lugar, una mayor respuesta dependiendo del destino del gasto. Así, encuentran que el impacto promedio de incrementar el IVA en $1 \%$ implica un aumento de alrededor de $\$ 5.747$ para alimentos y $\$ 4.771$ para celulares, alza que se ve compensada con una caída de $\$ 2.316$ en cultura y entretenimiento. Este impacto describe el promedio; no obstante, los autores descubren efectos diferenciados según el ingreso, lo cual es de gran pertinencia a la hora de dar argu- mentos empíricos a la posición que afirma la regresividad del IVA. El efecto, analizado desde la perspectiva de hogares con ingresos bajos, medios y altos se describe a continuación.

Tabla 1: Cambios en el gasto frente al IvA para 2007

\begin{tabular}{|l|l|l|l|}
\hline & Alimentos & $\begin{array}{c}\text { Cultura y } \\
\text { esparcimiento }\end{array}$ & Celulares \\
\hline $\begin{array}{l}\text { Ingresos } \\
\text { bajos }\end{array}$ & $\$ 5.443$ & $\$ 1.086$ & $\$ 51$ \\
\hline $\begin{array}{l}\text { Ingresos } \\
\text { medios }\end{array}$ & $\$ 3.858$ & $\$ 2.858$ & $\$ 3.533$ \\
\hline $\begin{array}{l}\text { Ingresos } \\
\text { altos }\end{array}$ & $\$ 7.427$ & $\$ 6.852$ & $\$ 15.192$ \\
\hline $\begin{array}{l}\text { Promedio } \\
\text { de hogares }\end{array}$ & $\$ 5.747$ & $\$ 2.316$ & $\$ 4.771$ \\
\hline
\end{tabular}

Tomado de Jaramillo \& Tovar, Incidencia del impuesto de valor agregado sobre los precios en Colombia, 2007b, pág. 19 y ss.

¿Qué significa eso en el caso concreto del estudio sobre los hogares colombianos? Que un aumento de un $1 \%$ en la tarifa de IVA sobre los productos mencionados aumenta el gasto en los diferentes rubros, independientemente del nivel de ingresos. No obstante, el aumento en este gasto extra se da de forma menos proporcional, puesto que representa una fracción mayor de un ingreso bajo que de un ingreso alto. Conclusión: el IvA afecta más a los hogares de ingresos bajos, sobre todo en alimentos y cultura.

\section{Sistema de exenciones y exclusiones}

Como se refirió anteriormente, Colombia tiene un sistema que comprende la categoría de bienes exentos y excluidos, según el cual para los primeros (exentos) contempla un mecanismo 
de devolución de impuestos pagados a lo largo de la cadena productiva. Este sistema en varias ocasiones ha sido criticado: la multiplicidad de tarifas y sistemas sería el origen de diversas ineficiencias y, a su vez, inequidades.

Así, se ha planteado que la heterogeneidad de regímenes es, en efecto, una fuente de distorsiones. En este sentido, nos remitimos de nuevo a lo expuesto acerca de la eficiencia y recordamos entonces que la neutralidad apunta a establecer una tributación que se distribuya de forma uniforme entre las diferentes actividades económicas. Con base en esto se ha señalado que esta multiplicidad impide la neutralidad. Por ello, una manera de aproximarnos a la inequidad surgida de la ineficiencia es ver cómo la forma de producción variará sustancialmente dependiendo de si el bien final de que se trata es excluido, exento o plenamente gravado.

En particular, se ha señalado el caso de los alimentos agrícolas. Al estar excluidos, el pago del IVA causado durante su producción se ve reflejado necesariamente en el precio, con lo que no solo "se reduce en mucho el supuesto efecto progresivo de la exclusión de alimentos" (Perry, 2010, pág. 18), sino que también se disminuye la potencial competitividad de sectores específicos. Vista así, la eficiencia no solo es un problema académico: la distorsión generada a partir del régimen actual incluso ataca el mismo propósito de equidad que las tarifas y regímenes diferenciales intentan establecer.

Adicionalmente, diferentes investigaciones y organismos han notado el problema de la multiplicidad y la cobertura de la base. La Dirección de
Impuestos y Aduanas Nacionales (DIAN) ha afirmado que las numerosas tarifas diferenciales han tenido el efecto de reducir de forma sustancial la base gravable del iva y lo han dejado en un 52\% (DIAN, 2009).

Ciertamente hay consideraciones de equidad al momento de definir los bienes a los que se les podrán descontar los impuestos pagados a lo largo de su producción. No obstante, también se ha señalado que la inclusión de unos u otros productos dentro de categorías más favorables muchas veces no ha respondido exclusivamente a razones de equidad sino también a diferentes consideraciones económicas (Perry, 2010).

Colombia es el único país de América Latina que no tiene una o dos tasas diferenciales (Perry, 2010). La comparación con países similares de América Latina, tales como Perú o Ecuador, muestra que habría espacio para que Colombia inicie efectivamente una reducción de tarifas y sistemas diferenciales sin un detrimento radical en equidad.

Una tercera forma de aproximarse al problema de inequidad generado a partir de la ineficiencia se da por cuenta del posible nexo causal entre complejidad jurídica de la estructura tributaria y el espacio dejado a la evasión e informalidad. La dificultad para administrar y recaudar los recursos surgidos de un sistema heterogéneo da lugar a inconvenientes de control (DIAN, 2012). Adicionalmente, se pone en desventaja a los pequeños productores, para quienes los costos de cumplir con el pago del gravamen se convierten en una carga proporcionalmente mayor que para las grandes empresas (McGee, 2008). 
Esto incide en la informalidad y evasión de esta obligación tributaria, lo cual tiene como resultado que el efecto de exclusiones y evasión sea una pérdida de recaudo que podría ascender a $-1.5 \%$ del PIB por cuenta de la informalidad y la evasión, y a -9.7 del PIB por exclusiones (DIAN, 2012).

Al hablar de la heterogeneidad de tarifas en el IVA, un punto que puede resultar de importancia a mediano o largo plazo es el cronograma de unificación planteado por la Comunidad Andina de Naciones (CAN). Este plan de integración de mercados se introdujo mediante la Decisión 599 de 2004, que establece parámetros y plazos para la unificación de los diferentes elementos de la obligación tributaria. Aunque su rango de modificaciones es amplio, expondremos dos elementos pertinentes a la luz del problema de la expansión de la base gravable hacia productos de consumo diario.

La primera de estas modificaciones es la eliminación de la categoría de bienes excluidos, en un lapso de diez años desde su entrada en vigencia, con excepciones taxativas en las importaciones de salud, educación y uso gubernamental. Al entrar en vigor esta disposición en nuestro país, tanto alimentos, maquinaria agrícola así como importaciones de tecnología o libros pasarían a tener una tarifa plena (CAN, 2008).

La segunda modificación que plantea un reto al sistema, con los presupuestos de equidad que existen hoy, es la eliminación de tarifas diferenciales para los bienes excluidos, salvo para bienes y servicios exportados, meta que se debería cumplir a cinco años de la vigencia de la Deci- sión. Esto plantea un cambio radical frente al régimen actual. Recordando lo mencionado en la sección sobre regulación del IVA, sacaría del régimen diferencial elementos de salud, educación, alimentos, etc. No obstante, la regla de tasa cero para exportaciones sería una de las directrices que ya está presente en la normatividad tributaria (CAN, 2008).

En síntesis, una eventual unificación conllevaría la homogeneización total del sistema tributario, no solo a nivel supranacional sino también en el ordenamiento interno. Por ejemplo, ordena la creación de una tasa general no mayor de 19\% y una preferencial no inferior al 5\% destinada a los bienes excluidos al momento de entrar en vigencia tal normatividad (CAN, 2008).

La finalidad de esta síntesis sobre los cambios que entrarían en vigor es poner de manifiesto la necesidad de discutir los efectos de una posible ampliación de la base y unificación de las tarifas, no solo como elemento coyuntural sino como compromiso internacional ya adquirido.

\section{B. Contexto internacional de la aplicación de impuestos de valor agregado: práctica en auge}

Esta sección aporta una descripción general de cómo ha sido la aplicación de impuestos de valor agregado alrededor del mundo, haciendo primero una breve contextualización y posteriormente puntualizando casos relevantes para Colombia, tales como los de España y Chile.

Los primeros impuestos a las ventas fueron introducidos en países europeos en los años cin- 
cuenta y sesenta. A partir de ahí, la tendencia clara ha sido la introducción de la tributación indirecta en la mayoría de países, proceso que se aceleró en la década de los noventa. Por lo tanto, su aplicación se da hoy en una amplia gama de países con variados niveles de ingreso, actividades y distribución. Se calcula que alrededor de 168 países ya han establecido impuestos de valor agregado. Un argumento de su éxito ha sido el que solo cinco países de quienes lo implementaron lo han retirado, cuatro de los cuales han regresado a este. (Lejeune, Daou-Azzi, \& Powell, 2009)

¿Qué podría explicar el éxito de los impuestos de valor agregado dentro de las agendas tributarias de tal diversidad de países? Varios factores han jugado a favor de una extensa implementación de impuestos a las ventas, según Bird (2005). En primer lugar, el haber sido establecidos como parte de un paquete de medidas de estabilización fiscal a cargo de organismos multilaterales como el Fondo Monetario Internacional o el Banco Mundial. En segundo lugar, el hecho de constituir uno de los pasos a seguir en los procesos de integración nacional. Ejemplo de ello son: la Unión Europea, que desde la Sexta Directiva de 1977 lo estableció como requisito para el ingreso a esta organización y donde a pesar de que persisten algunas diferencias relativas a bases y tarifas entre los países miembros, la tendencia hacia la unificación europea del impuesto de valor agregado ha sido observada como un modelo de integración supranacional; en la Comunidad Andina también ha sido puesto sobre la mesa como un eventual paso para la integración, según se expuso en el aparte anterior.
Aunque no hay un consenso extendido sobre sus efectos sobre el ciclo económico, sin embargo, como parte de las "bondades" de la implementación de sistemas de valor agregado se ha afirmado que puede constituirse en una herramienta de recaudo que adicionalmente impulse el crecimiento económico y genere nuevos empleos (Comisión Europea, 2006; ECD, 2008). La lógica detrás de esto estaría en eximir de una mayor carga fiscal a los tradicionales generadores de riqueza, tales como personas naturales y empresas, al igual que el ahorro e inversión.

Adicionalmente, se le ha atribuido a la tributación indirecta generar menos obstáculos políticos. Ciertamente, parece tener menos oposición legislativa la introducción de estos gravámenes o, al menos, grupos de presión menos organizados, que cuando se trata de un aumento de impuestos dirigidos a ingreso o beneficios (McGee, 2008).

Todo esto conduce a la aparente paradoja que contrapone la extensa cobertura de los impuestos de valor agregado a su poca capacidad redistributiva. Esta contradicción se ve acentuada por autores que señalan que los países más garantistas, por ejemplo los países históricamente inclinados hacia la social democracia, son los que acuden en mayor grado a la tributación indirecta, a pesar de ser un gravamen sobre la población que pretende ser el centro de las políticas de este tipo de Estados (Beramendi \& Rueda, 2007).

¿Podría decirse entonces que existe necesariamente una relación entre tarifas más altas y pérdida de bienestar en general para la sociedad? 
En el marco de esta pregunta se pasa a exponer brevemente el caso de dos países cuya pertinencia radica en el hecho de tener una estructura tributaria similar (España) o haberse constituido en punto de referencia a la hora de decidir la dirección de la política tributaria (Chile).

Veamos en primer lugar el caso de Chile, convertido en país modelo a nivel latinoamericano. En temas tributarios no es la excepción. En esta línea, se plantea como modelo económico, entre otras materias, de tributación.

La experiencia de Chile puede ser de alguna forma asimilable a Colombia. En 1975 estableció un sistema que genera tal obligación en cada fase de la producción, con derecho a la reposición del IVA pagado en los insumos del bien final -similar a Colombia- (ucCS AMERICA, 2012). Desde su establecimiento, el Iva ha adquirido de forma progresiva una preponderancia en el recaudo, llegando a convertirse en el $8.8 \%$ del PIB chileno -en Colombia su aporte es del 5.9\%(Clavijo, 2005). Adicionalmente, se ha convertido en la principal fuente de ingresos fiscales en Chile con 47\% (DIAN, 2012), lo cual pone de manifiesto la relevancia de la adecuada y eficiente administración del IVA en un modelo latinoamericano exitoso.

Además, tiene varios puntos que explican, entre otras cosas, su alto nivel de recaudación. Cabe mencionar, por ejemplo, la baja tasa de evasión tributaria en el cobro específico del impuesto de IVA. Otro elemento que apunta hacia la fortaleza del Iva en el caso chileno es la existencia de una tasa general uniforme de $18 \%$ desde 1998 y 19\% desde 2003, aplicada sobre todos los pro- ductos y la ausencia de exenciones, salvo para actividades relacionadas con comercio exterior (KPMG, 2011). Adicionalmente, Chile cuenta con impuestos sobre bienes suntuarios, que se aplican sobre transacciones de joyas, oro, alfombras y demás bienes calificados como de lujo por el Servicio de Impuestos Internos (UcCS AMÉRICA, 2012). Los bienes mencionados pagan una tarifa adicional del 15\%; algo similar propone para Colombia el proyecto de ley 166 de 2012.

En todo caso, cabe mencionar que estos mayores ingresos no vienen de manera exclusiva por cuenta de mayores tarifas. Se ven reforzados por una segunda razón que apunta hacia la fortaleza de este impuesto. Las tarifas uniformes, junto con reformas tendientes a la efectividad institucional, han aumentado de forma sustancial la capacidad jurídica de recaudar el IVA (Bergson, 2003). En el caso chileno, la evasión del pago del Iva reduce el recaudo potencial en 0, 9\% del PIB -por debajo del $1.5 \%$ en el caso colombiano, de acuerdo con estimaciones de la DIAN (2012)-.

España estableció también un sistema de IVA que se causa en cada fase de producción, con derecho a descontarlo al final de la cadena productiva. Este impuesto lo introdujo en 1985, en consonancia con las exigencias de la Sexta Directiva de 1977 de la Unión Europea, orientadas a la integración económica y a armonizar al máximo los mercados y regulaciones de los Estados miembros. Entre los principales lineamientos de la Directiva está la imposición del IVA sobre las transacciones realizadas, sin mayores especificaciones sobre bienes excluidos. Contempla una tarifa general mínima de 15\% 
y la autorización de uno o dos tipos reducidos para bienes de interés social o cultural, lista de bienes que se revisa con periodicidad. La aplicación del IVA ha sido de tal importancia dentro del proceso de integración europeo, que incluso la Comisión Europea está en capacidad de traer un país miembro ante el Tribunal Europeo de Justicia cuando considere que infringe el principio de neutralidad (Dale \& Nieuwenhuizen, 2009). Esto pone de manifiesto la viabilidad del control del impacto de los impuestos y, en particular, sus propiedades de neutralidad en una instancia judicial.

Este país ha establecido tres tipos de tarifas preferenciales intentando reintroducir equidad: general, reducido y superreducido, dependiendo del nivel de impacto que pueda tener el gravamen sobre el consumo. El tipo general es residual y aplica a todo aquel producto o servicio que explícitamente no se encuentre dentro de las categorías de bienes beneficiarios de la tarifa reducida o superreducida (Cabrera \& Cabrera, 2008). El superreducido aplica a alimentos de primera necesidad, tales como pan, leche, libros educativos, medicinas y algunos tratamientos, vehículos para la movilidad de personas con discapacidad y otros productos destinados a personas de este grupo. El tipo reducido se aplica sobre el consumo de agua en cualquier forma, otros alimentos, productos destinados a diagnóstico, venta de vivienda destinada a trabajo, entre otros (Cabrera \& Cabrera, 2008). El mantener tales tarifas le ha valido algunos reclamos por parte de la Comisión Europea. En 2010 esta autoridad reclamó la no observancia de la Sexta Directiva Europea, considerando que los servicios médicos no pueden recibir ta- sas diferenciales, salvo para personas con discapacidad (KPMG, 2011).

Si bien la base del sistema español como tal no ha sufrido variaciones sustanciales, las tarifas sí, y es ahí donde recientemente y de manera clara han tomado fuerza los cuestionamientos acerca de la equidad en la tributación indirecta. La variación de tarifas para los tres tipos: general, reducido y súper-reducido ha pasado de $15 \%, 6 \%$ y $4 \%$ en 1993 a 16\%, 7\% y 4\% en 2008, respectivamente (Cabrera \& Cabrera, 2008) y desde septiembre de 2012, las tasas respectivas son de $21 \%, 10 \%$ y $4 \%$.

El aumento de tales tarifas ha estado fuertemente atado a la polémica sobre su incidencia en el consumo diario de las personas. Si bien el tipo superreducido se ha mantenido sin alteraciones durante los últimos casi veinte años, el impacto de la última reforma ha sido plausible en lo que lleva de vigencia. Sin embargo, algunas estimaciones apuntan hacia reducciones drásticas en consumo de otros productos en el mes de vigencia de la tarifa general y reducida (The Wall Street Journal, 2012; The New York Times, 2012). Lo anterior podría atarse al aumento de tarifas diseñado en aras de lograr austeridad y, por lo tanto, a la ausencia de medidas compensatorias de las tarifas más altas. Las reformas mencionadas carecen de los elementos que precisamente se suelen asociar con medidas graduales que minimicen el impacto a corto plazo de estas. En todo caso, España pasó de ser uno de los regímenes fiscales más laxos de la Unión Europea cuando tenía la tarifa del 18\% (Pricewaterhouse Coopers, 2010) a tener la octava tarifa más alta tras las reformas. 


\section{CONCLUSIONES: POSIBLES} LINEAMIENTOS

Esta investigación ha tenido como objetivo la delimitación del principio constitucional de la eficiencia en materia tributaria, para lo cual ha tomado tres puntos principales: primero, mostrar la regulación constitucional y legal del iva bajo el principio constitucional de eficiencia; segundo, indicar qué debe tener el contenido óptimo de la eficiencia económica y cómo esto se contrapone, bajo alguna perspectiva, con el principio constitucional de equidad; y por último, mostrar las incidencias prácticas que este conflicto ha tenido a nivel nacional e internacional. Teniendo en mente esto, es posible ahora establecer ciertos lineamientos que de alguna forma hacen menos antagónicos los elementos que estarían detrás del principio de eficiencia de los impuestos indirectos con estándares que exige el principio de equidad.

Dos rasgos que son atribuidos a los impuestos indirectos eficientes tales como el IVA son: su implementación sobre una base amplia y una tarifa única o con el menor número de estas, características que les permiten encauzar esta eficiencia en el principio de neutralidad. Tal es la posición de organismos multilaterales, organizaciones supranacionales y círculos académicos, quienes sostienen que esta es una vía para generar unas mínimas variaciones de precios. De igual forma resulta ser la posición de los países a los que se les reconocen reformas tributarias exitosas: Chile o la Unión Europea. Esta posición, en todo caso, pasa por gravar varios bienes considerados en la llamada canasta fa- miliar y ha traído aparejadas objeciones sobre la equidad, puesto que por un lado equivale a imponer cargas sobre el consumo de elementos que no pueden entenderse como manifestaciones de riqueza por ser elementos de la canasta familiar cuyo gasto resulta ineludible y, por otro lado, constituye un gravamen que afecta proporcionalmente más a los hogares de menos ingresos. Ante tales perspectivas, ¿por qué vía puede armonizarse el hecho de que los impuestos de IVA tengan una extensa implementación a pesar de estas objeciones?

Un camino que puede lograrlo es observar los dos rasgos anteriores como puntos integrantes de un objetivo más general, que alcance la regulación por medio de reglas claras y simples, lo cual no es irrelevante. Por el contrario, a partir de la experiencia de Chile se ve posible lograr simultáneamente mayor eficiencia y mejores niveles de cumplimiento. Con estas dos condiciones como reglas claras, se obtiene la eficiencia económica que en el tema de tributación adopta la cara del principio de neutralidad.

Lo importante aquí es observar cómo la eficiencia, en este caso, resulta ser un vehículo potencial para lograr más equidad. Las vías por las que actúa están en dos frentes: por un lado, reducir las variaciones de precios que surgen en los bienes de la canasta familiar, distorsión que evidencia que el problema de la eficiencia tiene una clara incidencia sobre los bienes que el sistema tributario a priori desea proteger; y por otro, mejorar en recaudación y eficiencia para compensar los efectos que bases amplias y tarifas uniformes generen. Esto se explicaría por el aumento del recaudo: la reducción de ex- 
clusiones y evasión permitiría pasar el recaudo efectivo de $5.1 \%$ del PIB a cerca del 16\% (DIAN, 2012). El aumento del recaudo permite la discusión sobre la viabilidad de tales medidas compensatorias. Dichas medidas constituyen un punto de consenso entre diversas posiciones, entre las cuales cabe mencionar la de la sentencia C-776 de 2003 ${ }^{10}$; la de académicos en economía, por ejemplo Perry (2012), Clavijo (2005), Steiner (2012), entre otros y académicos especializados en derecho tributario (Uckmar, 2002; Plazas Vega, 2006, por citar algunos).

A partir de aquí es posible concluir, instando a observar la equidad o eficiencia del sistema tributario de una forma global: "si los impuestos con alícuota proporcional y su recaudación están destinados esencialmente a los sectores menor carenciados, puede sostenerse que se están cumpliendo los objetivos de la progresividad" (Ukmar, 2002, pág. 72)

No sería suficiente con tomar una foto parcial de este y contemplar qué nivel adoptan determinadas tarifas. Es necesario acudir a principios rectores de la tributación y evaluar qué incidencia tienen estos en las transacciones de los contribuyentes y qué destino se les darán. En el caso del IVA, las características de bases amplias y uniformidad de tarifas satisfacen el principio de eficiencia (entendiéndola bajo su acepción de neutralidad). Pero al mismo tiempo cumplen una función como instrumento hacia un sistema tributario potencialmente redistributivo, analizando qué reciben a cambio los contribuyentes.

10 Ver: Corte Constitucional, sentencia C-597 de 2000, M. P.: Álvaro Tafur Galvis.
Esto, por lo tanto, invita a analizar desde una perspectiva global y multidimensional la ampliación de la base y la creación de tarifas uniformes, anotando que un juicio sobre inequidad debe ir más allá de la norma en particular y más bien evaluar el impacto social de esta, proceder que vuelve más difuso el antagonismo entre los dos principios. Para concluir esta exposición sobre el principio de eficiencia, resaltando la necesidad de terminar tal antagonismo, es posible traer a consideración una frase del profesor Miguel Urrutia: “una buena macroeconomía es la mejor política social”"11.

\section{Bibliografía}

Acemoglu, D., Golosov, M., \& Tstrinski, A. (Mayo de 2008). Political Economy of Mechanisms. Econometrica, 76(3).

Arango, R. (2005). El concepto de derechos sociales fundamentales. Bogotá: Legis.

Atkinson, A. (Noviembre de 1977). Optimal Taxation and the Direct versus Indirect Tax Controversy. The Canadian Journal of Economics / Revue canadienne d'Economique, 10(4).

Beramendi, P., \& Rueda, D. (2007). Social Democracy Constrained: Indirect Taxation in Industrialized Democracies. British Journal of Political Science, 10(4).

Bergson, M. (Marzo de 2003). Tax Reforms and Tax Compliance: The Divergent Paths of Chile and Argentina. Journal of Latin American Studies, Cambridge University Press, 35(3).

11 Comunicación personal, 2011. 
BIRD, R. (2005). Value added taxes in developing and transitional countries: lessons and questions. Atlanta: Georgie State University. Working paper 05-05.

BIRD, R., \& GEndRon, P. (2005). The VAT in developing and transitional countries. Cambridge: Cambridge University Press.

Cabrera, J., \& Cabrera, M. (2008). Todo IVA 2008. Valencia: CISS, Grupo Wolters Kluwer.

Can. (24 de septiembre de 2008). Armonización para Colombia de las decisiones 59 y 600 de 2004: estudio comparativo. Recuperado en septiembre de 2012, de CAN: http://intranet.comunidadandina.org/Documentos/Reuniones/DTrabajo/SG_SEM_All_dt\%205.pdf

Castañeda, J. E. (2010). Aplicación del IVA en CoIombia: doctrina unificada de la DIAN. Bogotá: Estudios Fiscales Ltda.

Clavijo, S. (2005). Tributación, equidad y eficiencia: guía para salir de un sistema tributario amalgamado. Borradores de Economía no. 325. Bogotá: Banco de la República.

CNossen, S. (2009). A primer on VAT as perceived by lawyers, economists and accountants. En M. Lang, P. Melz, \& E. Kristofferson, Value Added Tax and Direct Taxation: similarities and differences. Amsterdam: IBFD.

Colombia. Constitución Política de 1991. (s.f.).

Colombia. Consejo de Estado, Sección Cuarta. (2009). Sentencia Rad. 11001-03-27-0002007-00048-00 (16873) del 26 de noviembre de 2009. C. P.: Héctor Romero Díaz.
Colombia. Corte Constitucional. (s.f.). Sentencia C-198 de 2012. M. P.: Nilson Pinilla.

Colombia. Corte Constitucional. (s.f.). Sentencia C-261 de 2002. M. P.: Clara Inés Vargas.

Colombia. Corte Constitucional. (s.f.). Sentencia C-776 de 2006. M. P.: Manuel José Cepeda.

Colombia. Corte Constitucional. (s.f.). Sentencia C-397 de 2011. M. P.: Jorge Ignacio Pretelt Chaljub.

Colombia. Corte Constitucional. Sentencia C-776 de 2003.

Colombia. Corte Constitucional. (s.f.). Sentencia C-341 de 1998. M. P.: José Gregorio Hernández.

Colombia. Corte Constitucional. (s.f.). Sentencia C-222 de 1995. M. P.: José Gregorio Hernández.

Colombia. Corte Constitucional. (s.f.). Sentencia C-173 de 2010. M. P.: Jorge Ignacio Pretelt Chaljub.

Colombia. Corte Constitucional. (s.f.). Sentencia C-597 de 2000. M. P.: Álvaro Tafur.

Comisión EuRopeA. (2006). Macroeconomic effects of a shift from direct to indirect taxation: a simulation for 15 EU member States. Recuperado el 2 de octubre de 2012, de Comisión Europea: http://www.oecd.org/tax/taxpolicyanalysis/39494151.pdf 
Cullis \& Jones. (1998). Public finance and public choice. Oxford: Oxford University Press.

Dale, S., \& Nieuwenhuizen, W. (2009). Value added tax yearbook: VAT decisions of the European Court of Justice 1974-2008. Alphen aan den Rijn: Kluwer Law International.

Dane. (2012). Indice de precios al consumidor: preguntas frecuentes. Recuperado el 15 de octubre de 2012, desde: http://www.dane. gov.co/files/faqs/faq_ipc.pdf

Dian. (2009). DIAN. Recuperado desde: www. dian.gov.co/descargas/Servicios/OEE-Documentos/Cuadernos/Evasion_IVA_en_Colombia_2000-2008.pdf

Dian. (Enero de 2012). Elementos para la discusión de una reforma del IVA en Colombia. Cuaderno de Trabajo No. 046. Recuperado el 14 de septiembre de 2012, desde: www. ceta.org.co/html/archivos/foro/ArchivoForolD831.pdf

ENGLISCH, J. (2009). VAT/GST and direct taxes: different purposes. En M. Lang, E. Kristofferson, \& P. Melz, Value Added Tax and Direct Taxation: similarities and differences. Amsterdam: IBFD.

Fergusson, L., \& Suárez, G. (2010). Política fiscal: un enfoque de tributación óptima. Bogotá: CEDE.

Hemels, S. (2009). Influence of different purposes of value added tax and personal income tax on an effective and efficient use of tax incentives: taking tax incentives for the arts and culture as an exemple. En M. Lang, P. Melz, \& E. Kristofferson, Value Added Tax and Direct Taxation: similarities and differences. Amsterdam: IBFD.

Jaramillo, C. \& Tovar, J. (2007). El impacto del impuesto de valor agregado sobre el gasto en Colombia. Bogotá: CEDE.

Jaramillo, C. \& Tovar, J. (2007). Incidencia del impuesto de valor agregado sobre los precios en Colombia. Bogotá: CEDE.

KPMG. (2011). KPMG. Recuperado el 14 de septiembre de 2012, de Corporate \& Indirect Tax Survey 2011: http://www.kpmg.com/ Global/en/IssuesAndInsights/ArticlesPublications/Documents/corporate-and-indirecttax-rate-survey-2011.pdf

Lejeune, I., Daou-Azzi, J. \& Powell, M. (2009). The balance has shifted to consumption taxeslessons learned and best practices for VAT. En M. Lang, P. Melz \& E. Kristofferson, Value Added Tax and Direct Taxation: similarities and differences. Amsterdam: IBFD.

Lewin Figueredo, A. (Diciembre de 15 de 2002b). La jurisprudencia de la Corte Constitucional en materia tributaria y su incidencia en la jurisprudencia del Consejo de Estado. Revista de Derecho Público (15).

Lewin Figueredo, A. (2002a). Principios constitucionales del derecho tributario: análisis de la jurisprudencia de la Corte Constitucional, 1992-2001. Bogotá: Universidad de los An- 
des, Fac. de Derecho: Instituto Colombiano de Derecho Tributario.

LipińSKA, A. \& Thadden, L. von. (2009). Monetary and fiscal effects of indirect tax changes in a currency union. Frankfurt: European Central Bank. Working paper series No. 1097.

LoRA, E. (2008). Técnicas de medición económica. Bogotá: Alfaomega.

MAYSHAR, J. (Septiembre de 1995). Dalton-Improving Indirect Tax Reform. The American Economic Review, 85(4).

McGee, R. (2008). Comparative study of indirect taxes in transition economies and the European Union. En R. McGee, Taxation and Public Finance in Transition Economies. New York: Springer.

Molter, L. C. (2012). Fiscal Policy in Colombia: Tapping Its Potential for a More Equitable Society. Washington: Policy Research Working Paper 6092. The World Bank: Latin America and the Caribbean Region Poverty Reduction and Economic Management Unit.

Nerudova, D. \& Siroki, J. (2009). The principle of neutrality: VAT/GST vs. Direct Taxation. En M. Lang, P. Melz \& E. Kristofferson, Value Added Tax and Direct Taxation: similarities and differences. Amsterdam: IBFD.

OECD. (2008). Do taxes structure affect aggregate economic growth? Empirical evidence from panel of OECD countries. Economics Department Working Paper No. 643.
Peralta, G. (2011). Criterios del análisis económico del derecho para la evaluación de normas jurídicas: el caso del IVA a las ventas de bienes corporales muebles en Colombia. Tesis. Universidad de los Andes.

Perry, G. (2010). Hacia una reforma tributaria estructural. Recuperado el 14 de septiembre de 2012, desde: http://www.fedesarrollo. org.co/wp-content/uploads/2011/08/impnalperry.pdf

Perry, G. (26 de mayo de 2012). Mitos Tributarios. El Tiempo.

Plazas Vega, M. (1989). El iva en Colombia. Bogotá: Temis.

Plazas Vega, M. (2006). Derecho de la hacienda pública y derecho tributario. Vol. II. Bogotá: Temis.

Pricewaterhouse Coopers. (2010). España, entre los países con un tipo de IVA más. Recuperado el 21 de noviembre de 2012, desde: http://www.pwc.es/es_es/es/sala-prensa/ assets/guia-iva-241110.pdf

Roemer, A. (2008). Introducción al análisis económico del derecho. México: Fondo de Cultura Económica.

Rubıo, M. (2007). Economía jurídica: introducción al análisis económico del derecho iberoamericano. Bogotá: Universidad Externado de Colombia.

Steiner, R. (21 de febrero de 2012). Focoeconómico. Recuperado el 27 de febrero 
de 2012, desde: http://focoeconomico. org/2012/02/21/consideraciones-en-tornoa-una-reforma-tributaria-integral/

Stiglitz, J. \& Emran, S. (2005). On selective indirect tax reform in developing countries. Journal of Public Economics (89).

Tarsitano, A. (2000). El principio de la realidad económica y el exceso de potestad calificadora del fisco. En R. Asorey. Protección constitucional de los contribuyentes. Madrid, Barcelona: Marcial Pons.

The New York Times. (15 de octube de 2012). Spain Waits, and Europe Frets. Recuperado el 20 de octubre de 2012, desde: http:// www.nytimes.com/2012/10/16/business/ global/spain-may-pay-price-for-delaying-aidrequest.html?pagewanted=all\&_r=0
The Wall Street Journal. (29 de octubre de 2012). Spain Retail Sales Fall at Record Pace. Recuperado el 30 de octubre de 2012, desde: http://online.wsj.com/article/SB10001424 05297020484050457808634002023378 4.html

Uccs America, Dinamarca, D. \& Castañeda, J. (2012). Impuestos en Chile. Recuperado el 10 de noviembre de 2012, desde: http://www.uccsamerica.org/espanol/Archivos/Biblioteca/ Impuestos\%20en\%20Chile.pdf

Ukmar, V. (2002). Principios comunes del derecho tributario. Bogotá: Temis.

Unión EUROPEA. (1977). Directiva 77/388/CEE del Consejo en materia de armonización de las legislaciones de los Estados miembros relativas a los impuestos sobre el volumen de negocios - Sistema común del Impuesto sobre el Valor Añadido: base imponible uniforme. 